\section{Deligne Awarded 2013 Abel Prize}

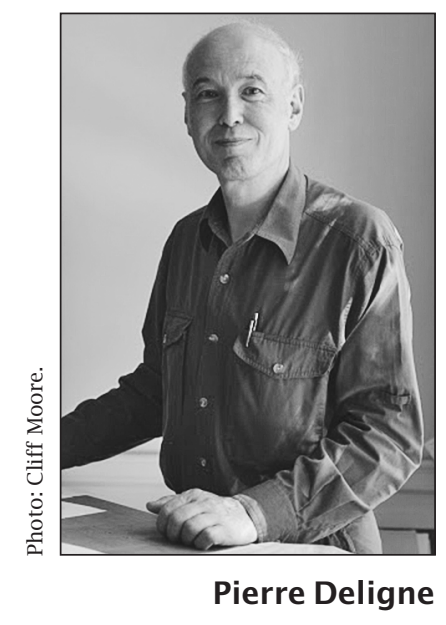

The Norwegian Academy of Science and Letters has awarded the Abel Prize for 2013 to PIERRE DELIGNE of the Institute for Advanced Study "for seminal contributions to algebraic geometry and for their transformative impact on number theory, representation theory, and related fields." The Abel Prize recognizes contributions of extraordinary depth and influence to the mathematical sciences and has been awarded annually since 2003. It carries a cash award of $6,000,000$ Norwegian kroner (approximately US $\$ 1$ million). Deligne received the Abel Prize in an award ceremony in Oslo, Norway, on May 21, 2013.

\section{Citation}

Geometric objects such as lines, circles, and spheres can be described by simple algebraic equations. The resulting fundamental connection between geometry and algebra led to the development of algebraic geometry, in which geometric methods are used to study solutions of polynomial equations and, conversely, algebraic techniques are applied to analyze geometric objects.

Over time, algebraic geometry has undergone several transformations and expansions and has become a central subject with deep connections to almost every area of mathematics. Pierre Deligne played a crucial role in many of these developments.

Deligne's best known achievement is his spectacular solution of the last and deepest of the Weil conjectures, namely the analogue of the Riemann hypothesis for algebraic varieties over a finite field. Weil envisioned that the proof of these conjectures

DOI: http://dx.doi.org/10.1090/noti1022 would require methods from algebraic topology. In this spirit, Grothendieck and his school developed the theory of $l$-adic cohomology, which would then become a basic tool in Deligne's proof. Deligne's brilliant work is a real tour de force and sheds new light on the cohomology of algebraic varieties. The Weil conjectures have many important applications in number theory, including the solution of the Ramanujan-Petersson conjecture and the estimation of exponential sums.

In a series of papers, Deligne showed that the cohomology of singular, noncompact varieties possesses a mixed Hodge structure that generalized the classical Hodge theory. The theory of mixed Hodge structures is now a basic and powerful tool in algebraic geometry and has yielded a deeper understanding of cohomology. It was also used by Cattani, Deligne, and Kaplan to prove an algebraicity theorem that provides strong evidence for the Hodge conjecture.

With Beilinson, Bernstein, and Gabber, Deligne made definitive contributions to the theory of perverse sheaves. This theory plays an important role in the recent proof of the fundamental lemma by Ngo. It was also used by Deligne himself to greatly clarify the nature of the Riemann-Hilbert correspondence, which extends Hilbert's 21st problem to higher dimensions. Deligne and Lusztig used l-adic cohomology to construct linear representations for general finite groups of Lie type. With Mumford, Deligne introduced the notion of an algebraic stack to prove that the moduli space of stable curves is compact. These and many other contributions have had a profound impact on algebraic geometry and related fields.

Deligne's powerful concepts, ideas, results, and methods continue to influence the development of algebraic geometry, as well as mathematics as a whole. 


\section{Biographical Sketch}

Pierre Deligne was born in 1944 in Brussels, Belgium. He is professor emeritus in the School of Mathematics at the Institute for Advanced Study (IAS), Princeton, New Jersey.

When Deligne was around twelve years of age, he started to read his brother's university math books and to demand explanations. His interest prompted a high school math teacher, J. Nijs, to lend him several volumes of Elements of Mathematics by Nicolas Bourbaki, the pseudonymous grey eminence that called for a renovation of French mathematics. This was not the kind of reading matter that one would normally dream of offering a fourteen-year-old, but for Deligne it became a life-changing experience. From then on he never looked back.

Although his father wanted him to become an engineer and to pursue a career that would afford him a good living, Deligne knew early on that he should do what he loved, and what he loved was mathematics. He studied mathematics at the Université Libre de Bruxelles (University of Brussels) and received his Licence en mathématiques, the equivalent of a B.A., in 1966 and his Ph.D., Doctorat en mathématiques, in 1968. In 1972 Deligne received the doctorat d'Etatés Sciences Mathématiques from Université Paris-Sud 11.

Deligne went to the University of Brussels with the ambition of becoming a high school teacher and of pursuing mathematics as a hobby for his own personal enjoyment. There, as a student of Jacques Tits, Deligne was pleased to discover that, as he says, "One could earn one's living by playing, i.e., by doing research in mathematics."

After a year at École Normale Supérieure in Paris as auditeur libre, Deligne was concurrently a junior scientist at the Belgian National Fund for Scientific Research and a guest at the IHES. He was a visiting member at IHES from 1968 to 1970 , at which time he was appointed its youngest permanent member.

Concurrently, he was a member (1972-1973, 1977) and visitor (1981) in the School of Mathematics at the IAS. He was appointed to a faculty position there in 1984 .

In 1974 he received the François Deruyts Prize of the Belgium Royal Academy and the Henri Poincaré Medal from the French Academy of Sciences. He received the A. De Leeuw-Damry-Bourlart Prize in 1975 from the Belgian National Science Foundation. In 1978 he was awarded the Fields Medal. In 1988 he and A. Grothendieck received the Crafoord Prize of the Royal Swedish Academy of Sciences. In 2004 he received the Balzan Prize in Mathematics, which carries a cash award of 1 million Swiss francs. Because the Balzan Foundation requires that half of the sum be spent to support young researchers, Deligne chose to establish three-year research grants to support the most active young mathematicians working in Russia, Ukraine, and
Belarus. In 2008 Deligne was awarded the Wolf Prize in Mathematics jointly with P. Griffiths and D. Mumford.

In 2006 Deligne was made a viscount by King Albert II of Belgium, and the Belgian postal service issued a postage stamp in honor of his achievements in fundamental mathematics.

He has been an honorary member of the Moscow Mathematical Society since 1995 and of the London Mathematical Society since 2003. He has been elected a foreign honorary member of the American Academy of Arts and Sciences (1978) and a foreign associate of the U.S. National Academy of Sciences (2007). In 2009 he became a member of the American Philosophical Society and a foreign member of the Royal Swedish Academy of Sciences.

\section{About the Prize}

The Niels Henrik Abel Memorial Fund was established in 2002 to award the Abel Prize for outstanding scientific work in the field of mathematics. The prize is awarded by the Norwegian Academy of Science and Letters, and the choice of Abel Laureate is based on the recommendation of the Abel Committee, which consists of five internationally recognized mathematicians.

Previous recipients of the Abel Prize are: JeanPierre Serre (2003), Michael Atiyah and I. M. Singer (2004), Peter Lax (2005), Lennart Carleson (2006), S. R. S. Varadhan (2007), John G. Thompson and Jacques Tits (2008), Mikhail L. Gromov (2009), John Tate (2010), John Milnor (2011), and Endre Szemerédi (2012).

-From an announcement of the Norwegian Academy of Science and Letters 